Published in final edited form as:

AIDS Behav. 2020 February ; 24(2): 532-539. doi:10.1007/s10461-019-02689-0.

\title{
Trends in HIV Testing Among US Adults, Aged 18-64 Years, 2011-2017
}

\author{
Deesha Patel ${ }^{1}$, Christopher H. Johnson ${ }^{1}$, Amy Krueger ${ }^{1}$, Barbara Maciak ${ }^{1}$, Lisa Belcher ${ }^{1}$, \\ Norma Harris ${ }^{1}$, Elizabeth A. DiNenno ${ }^{1}$ \\ ${ }^{1}$ Division of HIV/AIDS Prevention, National Center for HIV/AIDS, Viral Hepatitis, STD, and TB \\ Prevention, Centers for Disease Control and Prevention, Atlanta, GA, USA
}

\begin{abstract}
In 2006, Centers for Disease Control and Prevention (CDC) recommended HIV screening in healthcare or clinical settings for all persons aged 13-64 years and annual rescreening for populations at high risk for HIV. We used the Behavioral Risk Factor Surveillance System to describe the prevalence and trends of ever tested for HIV and tested for HIV in the past 12 months among US adults. The percentage of ever tested increased from $42.9 \%$ in 2011 to $45.9 \%$ in 2017; testing in the past 12 months increased from 13.2\% in 2011 to $14.8 \%$ in 2017. Despite these increases, less than half of US adults have ever been tested for HIV over ten years after CDC's recommendations. Increasing the prevalence of routine HIV screening and rescreening among individuals at high risk will reduce the number of undiagnosed persons with HIV infection and thus prevent new HIV infections-a key strategy in the Ending the HIV Epidemic initiative.
\end{abstract}

\section{Keywords}

HIV; Prevention; Testing; Screening; Trends

\section{Introduction}

Since 2013, the estimated number of new HIV infections in the United States has leveled off at about 39,000, with the highest number of new HIV infections among persons aged 25-34 years, blacks or African Americans (hereafter referred to as blacks), Hispanic/Latinos, and gay, bisexual, and other men who have sex with men (collectively referred to as MSM) in 2016 [1]. Knowledge of HIV status is important in order for persons to gain access to proper HIV medical care, which can improve quality of life, extend life expectancy, and modify behaviors that could prevent transmission to others [2]; however, about $14 \%$ of persons infected with HIV were unaware of their status in 2016 [1]. HIV testing identifies persons with HIV infection, allowing these individuals to be become aware of their HIV status and to seek timely and proper medical care and treatment, thus reducing adverse HIV-related health

Deesha Patel, DPatel3@cdc.gov.

Electronic supplementary material The online version of this article (https://doi.org/10.1007/s10461-019-02689-0) contains supplementary material, which is available to authorized users.

Publisher's Note Springer Nature remains neutral with regard to jurisdictional claims in published maps and institutional affiliations. 
outcomes. Furthermore, knowledge of HIV status is important for reducing HIV transmission, as approximately $40 \%$ of new HIV infections are transmitted by people who do not know they have the virus [3]. "Diagnosing all individuals with HIV as early as possible after infection" is one of the four pillars of the new Ending the HIV Epidemic initiative, which aims to reduce the number of new infections by $75 \%$ within five years and then by $90 \%$ within ten years [4].

HIV testing has long been an integral part of HIV prevention. In 2006, Centers for Disease Control and Prevention (CDC) released the Revised Recommendations for HIV Testing of Adults, Adolescents, and Pregnant Women in Health-Care Settings (hereafter referred to as revised recommendations). The revised recommendations recommend screening for HIV (i.e., testing at least once, regardless of clinical signs or symptoms) for all persons aged 1364 years, as well as rescreening at least annually for populations at high risk for HIV infection (i.e., people who inject drugs and their sex partners; people who exchange sex for money or drugs; sex partners of HIV-infected people; MSM; or heterosexuals who themselves or whose sex partners have had more than 1 sex partner since their most recent HIV test) [5]. Subsequent to these recommendations, CDC funded expanded testing initiatives in select health department jurisdictions in 2007 [6], followed by high-impact prevention programs beginning in 2012, which included increased funding for HIV testing in high-prevalence areas [7].

Earlier studies-with varying methodologies, age ranges, and time periods-have examined HIV testing prevalence and trends prior to and shortly after the revised recommendations. Among those aged 15-44 years, the National Survey of Family Growth (NSFG) found that $59 \%$ of women reported ever being tested for HIV in 2006-2010 compared with 55\% in 2002; among men, the percentage of ever tested decreased from $47 \%$ in 2002 to $42 \%$ in 2006-2010. In 2006-2010, 21\% of women and 13\% of men reported being tested in the past 12 months [8]. According to a CDC report examining 2010 National Health Interview Survey (NHIS) data, $45.0 \%$ of adults aged 18-64 years reported ever being tested for HIV and $10.1 \%$ were tested in the past 12 months. From 2000 to 2010, the percentage of ever tested increased significantly overall and among all race/ethnicity groups, persons aged 2564 years, and males and females; the percentage of tested in the past 12 months only increased significantly among blacks and Hispanic/Latinos and decreased significantly among whites and persons of other race/ethnicity [9]. Finally, another study, using 20032006 and 2007-2010 National Health and Nutrition Examination Survey (NHANES) data among adults aged 18-59 years, found statistically significant increases in percentage of ever tested for HIV among males, blacks, and persons aged 50-59 years, but the overall increase (2003-2006: 42.1\%; 2007-2010: 44.5\%) was only significant after adjusting for predictors in a multivariable model [10].

More recent studies, however, have assessed HIV testing among specific subpopulations [11, 12] or have provided aggregate data among the general population but have not examined trends in HIV testing over time [13, 14]. A systematic review examining studies from 2005 to 2014 found that $63 \%$ to $91 \%$ of MSM had been ever tested for HIV, but only $39 \%$ to $67 \%$ were being tested annually as per the revised recommendations [11]. Among younger populations, an average of $22 \%$ of high school students in 2005-2013 who ever had sexual 
intercourse and 33\% of young adults aged 18-24 years in 2011-2013 reported being ever tested for HIV [12]. The percentage of persons ever tested for HIV according to 2006-2016 General Social Survey (GSS) data was approximately 40\% [13], whereas 2011-2015 NSFG data found that $39 \%$ of women and 54\% of men had never been tested for HIV [14]. In the geographic areas that are the initial focus of the Ending the HIV Epidemic initiative [4], $46.9 \%$ of respondents were ever tested for HIV and $14.5 \%$ in the past 12 months in the 50 local jurisdictions; for the seven states, $35 \%$ of respondents were ever tested for HIV and $9.3 \%$ in the past 12 months [15].

Given the importance of HIV testing in the first pillar of the Ending the HIV Epidemic initiative [4], it is important to monitor HIV testing trends at the national level.

Understanding the trends of HIV testing will allow CDC to track progress of HIV testing in the period before and in the years following the implementation of the Ending the HIV Epidemic initiative [4], as well as within the context of the revised recommendations [5]. Thus, the purpose of this manuscript is to describe the prevalence and trends of ever tested for HIV and tested for HIV in the past 12 months in recent years among a large nationally representative sample of US adults.

\section{Methods \\ Data Source}

The Behavioral Risk Factor Surveillance System (BRFSS) is an annual cross-sectional survey among non-institutionalized US adults aged 18 years and older that collects data on health-related risk behaviors, chronic health conditions, and use of preventive services [16]. Respondents' data are weighted to generalize sample results to the population of noninstitutionalized adults and provide nationally representative estimates. Prior to 2011, BRFSS was conducted using Random Digit Dialing sampling of landline telephones only. Starting in 2011, BRFSS expanded coverage to include respondents who received $100 \%$ of their calls on cell phones. Due to this change in methodology, we did not analyze data prior to 2011. Despite this limitation, we determined BRFSS to be the best data source at this time to assess HIV testing and monitor trends among the general population [17], as it provides recent and annual data as well as adequate sample sizes.

\section{Inclusion Criteria}

This analysis used BRFSS data based on the following inclusion criteria. Firstly, only data from respondents aged 18-64 years were included, in order to align with the age group of the revised recommendations [5]. Secondly, respondents must have definitively answered "yes" or "no" for the question of whether they were ever tested for HIV ("Have you ever been tested for HIV? Do not count tests you may have had as part of a blood donation. Include testing fluid from your mouth."). Respondents with responses of “don't know/not sure" or no responses (i.e., not asked, missing, refused) were excluded from analysis, as to minimize underestimation of HIV testing and to be consistent with similar prior analyses [9, $10,13,15,18]$. The distribution of responses is provided in Supplementary Table I. For the purpose of this manuscript, HIV testing encompasses both routine screening and diagnostic or targeted testing. 
In order to determine whether respondents were tested in the past 12 months, respondents must have provided at least the year of their last test ("Not including blood donations, in what month and year was your last HIV test?"). If the year of the test was the year before the interview, June - as a mid-point to the calendar year-was imputed. This allowed for the assumptions that (1) among those not providing the month of the test (i.e., only providing year), the distribution of those tested in the past 12 months was the same as those not tested in the past 12 months; and (2) among those tested in the past 12 months, half of those tests occurred in the first six months of the year and the other half in the last six months of the year. Excluding respondents with missing month data (i.e., assuming respondents were not at all tested in the past 12 months) would have underestimated the outcome.

\section{Data Analysis}

We estimated the percentage of the US population ever tested for HIV and tested for HIV in the past 12 months during 2011-2017 overall and by race/ethnicity (white, black, Hispanic/ Latino, other race/ethnicity [i.e., Asian, American Indian/Alaska Native, Native Hawaiian/ Pacific Islander, multiracial, and other race]), age (18-24 years, 25-34 years, 35-44 years, 45-64 years), and sex (male, female).

We also estimated the percentage of ever tested for HIV and tested for HIV in the past 12 months among those with at least one HIV risk behavior in 2011, 2012, 2016, and 2017; the question was not asked in 2013, 2014, or 2015. In 2011 and 2012, 2.7\% and 2.9\%

(Supplementary Table I) of respondents, respectively, reported having at least one HIV risk behavior: using intravenous drugs in the past year; being treated for a sexually transmitted or venereal disease in the past year; giving or receiving money or drugs in exchange for sex in the past year; and/or having anal sex without a condom in the past year. In 2016, having four or more sex partners in the past year was added to the HIV risk behavior question; $4.9 \%$ of respondents reported having at least one HIV risk behavior. The HIV risk behavior question was further modified in 2017: injecting any drug other than those prescribed in the past year; being treated for a sexually transmitted disease or STD in the past year; giving or receiving money or drugs in exchange for sex in the past year; having anal sex without a condom in the past year; and/or having four or more sex partners in the past year. In 2017, 5.1\% of respondents reported having at least one HIV risk behavior.

We conducted analyses in SAS version 9.4 and SUDAAN to account for the complex sample design of BRFSS and to incorporate analysis weights provided for that purpose. We measured each outcome as percentage (weighted) of persons tested, with associated $95 \%$ confidence intervals (CIs). We also estimated population totals of persons tested by using the sum of the analysis weights.

We also examined HIV testing prevalences over time to observe whether trends increased, decreased, or remained stable. We used orthogonal contrasts in SUDAAN to calculate pvalues for linear trends for ever tested for HIV and tested for HIV in the past 12 months overall and by race/ethnicity, age, and sex. A significant linear trend indicated that testing increased or decreased over the years compared; a non-significant linear trend suggested stable prevalences or no clear pattern of change. We used a lower significance threshold of $p$ $<0.01$ due to the large sample sizes available for analysis. 
Given that the outcomes of ever tested for HIV and tested for HIV in the past 12 months were dichotomous, we calculated estimated annual percent change (EAPC) to quantify magnitude of linear trends. Using the assumption of linearity on the log scale (i.e., assuming a constant rate of change), the EAPC was calculated as the difference in the model-predicted testing prevalence between successive years, divided by baseline prevalence. This measure quantified the rate of change over time and indicated the direction of change: a positive EAPC corresponded to an increasing trend, while a negative EAPC corresponded to a decreasing trend; a slope of zero implied no change between the beginning and ending time points. Because the method of contrasts is generally considered a more robust way of assessing trends, we calculated EAPC only to quantify the magnitude of change (i.e., $p$ value refers to significance of linear trend and not EAPC). However, significance of trends generally leads to the same conclusions despite the different underlying assumptions of contrasts and EAPC, although the relationship between EAPC and p-values may not be monotonic (e.g., greater magnitude of EAPC may not necessarily result in a more significant p-value; this may be due to the differing sizes of subpopulations).

\section{Results}

\section{Ever Tested for HIV}

The percentage of persons reporting ever being tested for HIV increased significantly from $42.9 \%$ in 2011 to $45.9 \%$ in 2017 (Table 1; linear contrast $\mathrm{p}<0.001$; EAPC $=0.46$ ), indicating that an estimated 4,314,000 more persons reported being ever tested in 2017 compared to 2011 (Supplementary Table II).

By race/ethnicity, the percentage of ever tested for HIV in 2017 was highest among blacks (68.6\%), followed by Hispanic/Latinos (48.1\%), whites (41.6\%), and persons of other race/ ethnicity (38.4\%); the percentage of ever tested for HIV increased significantly from 2011 to 2017 among whites $(\mathrm{p}<0.001$; EAPC $=0.54)$ and Hispanic/Latinos $(\mathrm{p}=0.0012 ;$ EAPC $=$ 0.36). The percentage of ever tested for HIV in 2017 was highest among persons aged 35-44 years $(58.4 \%)$, followed by persons aged 25-34 years (55.4\%), persons aged 45-64 years (40.3\%), and persons aged 18-24 years (31.5\%); the percentage of ever tested for HIV from 2011 to 2017 increased significantly for persons aged 35-44 years $(\mathrm{p}<0.001 ;$ EAPC $=0.66)$ and 45-64 years $(\mathrm{p}<0.001$; EAPC $=1.17)$ and decreased significantly for persons aged $18-$ 24 years $(\mathrm{p}<0.001 ;$ EAPC $=-0.55)$ and $25-34$ years $(\mathrm{p}<0.001 ;$ EAPC $=-0.36)$. The percentage of ever tested in 2017 for HIV was higher among females (48.8\%) than males (42.9\%); the percentage of ever tested increased significantly for both sexes from 2011 to 2017 ( $p<0.001$ for both; EAPC $=0.47$ for females; EAPC $=0.44$ for males $)$.

The percentage of ever tested for HIV among those reporting at least one HIV risk behavior was $68.1 \%$ in 2011, 66.2\% in 2012, and 65.2\% in 2016 and 2017 (compared to $42.8 \%$ in $2011,41.3 \%$ in 2012, 42.3\% in 2016, and $44.2 \%$ in 2017 among those not reporting any HIV risk behaviors). 


\section{Tested for HIV in the Past 12 Months}

The percentage of persons reporting being tested for HIV in the past 12 months increased significantly from $13.2 \%$ in 2011 to $14.8 \%$ in 2017 (Table 2 ; $p<0.001$; EAPC $=0.23$ ), indicating that an estimated 2,035,000 more persons reported being tested in the past 12 months in 2017 compared to 2011 (Supplementary Table III).

By race/ethnicity, the percentage of tested for HIV in the past 12 months in 2017 was highest among blacks (34.1\%), followed by Hispanic/Latinos (17.6\%), persons of other race/ ethnicity (12.8\%), and whites (10.5\%); percentage of tested in past 12 months for HIV increased significantly from 2011 to 2017 for whites ( $<0.001$; EAPC $=0.20)$ and blacks $(\mathrm{p}=0.0039$; EAPC $=0.33)$. The percentage of tested for HIV in the past 12 months in 2017 was highest among persons aged 25-34 years (22.3\%), followed by persons aged 18-24 years $(18.9 \%)$, persons aged 35-44 years (16.1\%), and persons aged 45-64 years (8.6\%); from 2011 to 2017, percentage of tested for HIV in the past 12 months increased significantly for persons aged 35-44 years $(\mathrm{p}<0.001$; EAPC $=0.45)$ and 45-64 years $(\mathrm{p}<$ $0.001 ; \mathrm{EAPC}=0.29)$ and decreased significantly for persons aged 18-24 years $(\mathrm{p}=0.0027$; $\mathrm{EAPC}=-0.28)$ and $25-34$ years $(\mathrm{p}=0.007 ; \mathrm{EAPC}=-0.19)$. The percentage of tested for HIV in the past 12 months in 2017 was higher among females (15.6\%) than males (14.0\%); percentage of tested in the past 12 months increased significantly from 2011 to 2017 for both sexes ( $\mathrm{p}<0.001$ for both; $\mathrm{EAPC}=0.24$ for females; $\mathrm{EAPC}=0.22$ for males).

The percentage of tested for HIV in the past 12 months among those reporting at least one HIV risk behavior was $32.8 \%$ in $2011,32.3 \%$ in $2012,32.9 \%$ in 2016, and $34.2 \%$ in 2017 (compared to $12.7 \%$ in 2011, 11.7\% in 2012, 11.5\% in 2016, and $13.1 \%$ in 2017 among those not reporting any HIV risk behaviors).

\section{Discussion}

From 2011 to 2017, the prevalence of persons aged 18-64 years in the United States reporting ever being tested for HIV increased from $42.9 \%$ to $45.9 \%$, resulting in an estimate of 4.3 million more persons being ever tested in 2017 compared to 2011. The prevalence of ever tested for HIV also increased during 2011-2017 for whites and Hispanic/Latinos; persons aged 35-44 years and 45-64 years; and both males and females. Approximately two-thirds of persons reporting at least one HIV risk behavior had been ever tested for HIV. The prevalence of ever tested for HIV decreased over time among those aged 18-24 years and 25-34 years, despite the suggestion that the optimal age for HIV screening among adolescents and young adults without identified risk factors is 25 years [19]. This decrease, coupled with a concern that young people have low awareness about advances in HIV prevention and treatment and misperceptions about HIV transmission [20], may indicate a potential risk for increased HIV infections and transmission in this population.

Despite the percentage increases of ever tested overall and in most subpopulations, the improvement of HIV testing in the general population over time has been suboptimal, especially in comparison to prior national studies with varying methodologies [8-10] that were conducted before or shortly after the revised recommendations [5]. Less than half of US adults aged 18-64 years reported ever being tested for HIV in accordance with the 
revised recommendations [5], suggesting that missed opportunities for HIV screening remain. Among the 39,720 persons with HIV infection diagnosed in 2015, the estimated median diagnosis delay was 3 years [21]. Among MSM and persons who inject drugs, about half of those who were unaware of their HIV infection had reported not being offered HIV testing despite visiting a clinician in the past year [22].

The percentages of ever tested among those reporting at least one HIV risk behavior were slightly higher in 2011 and 2012 than in 2016 and 2017, even though the prevalence of reporting at least one HIV risk behavior was higher in 2016 and 2017 (likely due to an additional response component). To date, all states but one have implemented opt-out HIV testing, which eliminates separate written consent for HIV testing and instead notifies patients of HIV testing under a general informed consent for medical care [23, 24]. Thus, it is possible that persons were not aware they were being tested for HIV in the more recent years, resulting in the slightly lower percentages of ever tested in the later years among those reporting at least one HIV risk behavior, especially since this is a population where HIV testing was more prevalent to begin with.

The prevalence of being tested for HIV in the past 12 months overall increased from 13.2\% in 2011 to $14.8 \%$ in 2017, with about one-third of those reporting at least one HIV risk behavior having been tested for HIV in the past 12 months. Although we could not assess the changes in percentage of testing for HIV in the past 12 months nationally over time among individual populations at high risk for HIV infection (e.g., MSM) with BRFSS data, our findings are consistent with data from GSS [13], which found that less than $50 \%$ of persons at high risk for HIV were tested for HIV in the past year-despite the recommendation that these persons should be screened at least annually [5]. CDC recently updated guidelines for screening MSM, suggesting that although sexually active MSM should be screened at least annually, clinicians can consider the potential benefits of more frequent HIV testing (e.g., every 3 or 6 months) for some asymptomatic sexually active MSM based on their individual risk factors, local HIV epidemiology, and local policies [11]. Certain jurisdictions, such as Seattle/King County, New York City, and San Francisco, have already implemented more frequent screening for MSM [11].

To support implementation of the revised recommendations [5] and help address the goals of the Ending the HIV Epidemic initiative [4], more routine HIV screening needs to be conducted in the United States. Routine HIV screening is a vital part of HIV prevention because it reaches persons who would not have been otherwise tested based on risk behaviors or provider assessment, is typically less costly than targeted testing (as it can be added to other laboratory tests during a clinical visit), results in considerably higher number of individuals tested and diagnosed, and helps to reduce the stigma of HIV testing [5]. However, targeted testing is still needed to reach persons who do not have access to or are less likely to access the healthcare system (e.g., youth, racial/ethnic minorities). Better understanding is needed to determine the appropriate combination of routine screening and targeted testing in communities based on their local epidemiology and available infrastructure, which would help to direct funding to programs accordingly. 
Healthcare providers can continue to strengthen HIV screening efforts among all persons in clinical settings and continue rescreening efforts among persons at high risk for HIV. Healthcare providers can incorporate HIV testing for all persons and for persons at high risk into standard clinical practice through institutional policy changes, electronic health record prompts, and provider education [25, 26]. In non-clinical settings, public health practitioners can expand access to HIV testing, especially among populations at high risk for HIV, through strategies such as social marketing and networking and couples-based HIV testing and counseling [27], as well as promoting home testing [28]. Funding opportunities, such as CDC's Integrated HIV Surveillance and Prevention Programs for Health Departments [29], support HIV screening efforts throughout the United States, especially among populations at high risk for HIV. Increasing the prevalence of HIV screening for both the general population and populations at high risk for HIV will help to reduce diagnosis delays, improve linkage to care, and reduce HIV transmission [2, 5].

This analysis is subject to a few limitations. Although BRFSS contained a question regarding HIV risk behaviors, it was not asked annually, response components varied over time, and we were not able to assess HIV testing nationally by individual populations at high risk for HIV because the HIV risk behavior question could not be stratified by its components and because the core BRFSS questionnaire did not include relevant questions (i.e., questions about sexual orientation and gender identity are part of an optional module). Another limitation to this analysis was the potential for sample bias since certain populations that might be at high risk for HIV [30, 31] may not have been surveyed; homeless persons [30] may not have access to cell phones and incarcerated persons [31] are not included in the sampling of the survey. Recall bias given the self-report nature of the survey was another limitation. The latter limitations, in combination with the implementation of opt-out testing [23, 24], could have underestimated the percentages of ever tested for HIV and tested for HIV in the past 12 months.

Since ever being tested can change in only one direction (i.e., from never to ever), it could be expected that the prevalence would steadily increase every year. However, because our study population was adults aged 18 to 64 years for repeated cross-sectional surveys, the cohort changed as individuals aged into or out of it. Deaths and in- and out-migration would have also affected the cohort. If younger persons (i.e., those coming into the cohort) test at higher rates than older persons (i.e., those aging out of it), this would tend to increase the prevalence of ever tested; if that is not the case, then the prevalence would tend to remain stable (or potentially even decrease). In our analysis, we found a statistically significant change in prevalence over time, but the absolute difference in percentages was not large (i.e., three percentage points). Similar considerations apply when one examines testing by age group since individuals age into and out of these cohorts and are defined by narrower age ranges. For our results, it is possible that the slight increase in ever being tested overall was because the decline in ever being tested among the entrants into the cohort was not as large as the increase in ever being tested among those remaining in the cohort.

Finally, because of changes in BRFSS methodology, we were not able to assess HIV testing prior to 2011. However, in comparison to other nationally representative surveys, we determined BRFSS to be the best data source at this time for assessing HIV testing trends 
among the general population. NHIS changed the location and context of its HIV-related questions in 2011, resulting in variability of estimates before and after 2011 [17], and NHANES and GSS have smaller sample sizes, as does NSFG, which is further limited to the age range of 15-44 years. However, it is possible that the statistically significant results in this analysis may have been driven by the large sample sizes available in BRFSS.

\section{Conclusions}

Over ten years after CDC's revised recommendations of HIV screening at least once in a lifetime for all persons aged 13-64 years and at least annually for those at high risk [5], less than half of US adults reported being ever tested for HIV and only about $15 \%$ were tested in the past 12 months. This suboptimal improvement in comparison to prior studies examining testing trends before or shortly after the revised recommendations [8-10], in addition to the many missed opportunities for HIV screening that have been documented elsewhere [21, 22], suggest that increasing the prevalence of HIV screening for both the general population and populations at high risk for HIV would help to achieve the Ending the HIV Epidemic initiative's first pillar of diagnosing persons with HIV infection as early as possible [4]. This would then reduce diagnosis delays, improve linkage to care and treatment, reduce adverse HIV-related health outcomes, and decrease HIV transmission [21].

\section{Supplementary Material}

Refer to Web version on PubMed Central for supplementary material.

\section{Acknowledgments}

Disclaimer The findings and conclusions in this article are those of the authors and do not necessarily represent the official position of the Centers for Disease Control and Prevention.

\section{References}

1. Centers for Disease Control and Prevention. Estimated HIV incidence and prevalence in the United States, 2010-2016. HIV Surveillance Supplemental Report 2019;24(No. 1). http://www.cdc.gov/hiv/ library/reports/hiv-surveillance.html. Accessed 4 Mar 2019.

2. White House Office of National AIDS Policy. National HIV/AIDS strategy for the United States: updated to 2020. https://files.hiv.gov/s3fs-public/nhas-update.pdf. Accessed 29 Jan 2019.

3. Gopalappa C, Farnham PG, Chen YH, Sansom SL. Progression and transmission of HIV/AIDS (PATH 2.0). Med Decis Mak. 2017;37(2):224-33.

4. Fauci AS, Redfield RR, Sigounas G, Weahkee MD, Giroir BP. Ending the HIV epidemic: a plan for the United States. JAMA. 2019;321(9):844-5. [PubMed: 30730529]

5. Branson BM, Handsfield HH, Lampe MA, et al. Revised recommendations for HIV testing of adults, adolescents, and pregnant women in health-care settings. MMWR Recomm Rep. 2006;55(RR-14):1-17.

6. Centers for Disease Control and Prevention. Expanded testing initiative. https://www.cdc.gov/hiv/ policies/eti.html. Accessed 28 Oct 2018.

7. Centers for Disease Control and Prevention. High-impact HIV prevention: CDC's approach to reducing HIV infections in United States. https://www.cdc.gov/hiv/pdf/ policies_NHPC_Booklet.pdf. Accessed 15 Jan 2018. 
8. Chandra A, Billioux VG, Copen CE, Balaji A, DiNenno E. HIV testing in the U.S. household population aged 15-44: data from the National Survey of Family Growth, 2006-2010. Natl Health Stat Rep. 2012;58:1-26.

9. Centers for Disease Control and Prevention. HIV testing trends in the United States, 2000-2011. Atlanta, GA: U.S. Department of Health and Human Services, Centers for Disease Control and Prevention; 2013.

10. Woodring JV, Kruszon-Moran D, Oster AM, McQuillan GM. Did CDC's 2006 revised HIV testing recommendations make a difference? Evaluation of HIV testing in the US household population, 2003-2010. J Acquir Immune Defic Syndr. 2014;67(3):331-40. [PubMed: 25153918]

11. DiNenno EA, Prejean J, Irwin K, et al. Evaluating the evidence for more frequent than annual HIV Screening of gay, bisexual and other men who have sex with men in the United States: results from a systematic review and CDC Expert Consultation. Public Health Rep. 2017;133(1):3-21. [PubMed: 29182894]

12. Van Handel M, Kann L, Olsen EO, Dietz P. HIV testing among US high school students and young adults. Pediatrics. 2016;137(2):e20152700. [PubMed: 26787047]

13. Pitasi MA, Delaney KP, Oraka E, et al. Interval since last HIV test for men and women with recent risk for HIV infection-United States, 2006-2016. MMWR Morb Mortal Wkly Rep. 2018;67(24): 677-81. [PubMed: 29927906]

14. Febo-Vazquez I, Copen CE, Daugherty J. Main reasons for never testing for HIV among women and men aged 15-44 in the United States, 2011-2015. Natl Health Stat Rep. 2018;107:1-12.

15. Pitasi MA, Delaney KP, Brooks JT, DiNenno EA, Johnson SD, Prejean J. HIV testing in 50 local jurisdictions accounting for the majority of new HIV diagnoses and seven states with disproportionate occurrence of HIV in rural areas, 2016-2017. MMWR Morb Mortal Wkly Rep. 2019;68(25):561-7.

16. Centers for Disease Control and Prevention. Behavioral Risk Factor Surveillance System. https:// www.cdc.gov/brfss/. Accessed 30 Apr 2018.

17. Van Handel MM, Branson BM. Monitoring HIV testing in the United States: consequences of methodology changes to national surveys. PLoS ONE. 2015;10(4):e0125.

18. Tan C, Van Handel M, Johnson C, Dietz P. HIV testing in publicly funded settings, National Health Interview Survey, 2003-2010. Public Health Rep. 2016;131(1):137-44. [PubMed: 26843679]

19. Neilan AM, Dunville R, Ocfemia MCB, et al. The optimal age for screening adolescents and young adults without identified risk factors for HIV. J Adolesc Health. 2018;62(1):22-8. [PubMed: 29273141]

20. Kaiser Family Foundation. National Survey of Young adults on HIV/AIDS. https://www.kff.org/ hivaids/report/national-survey-of-young-adults-on-hiv-aids/. Accessed 29 Oct 2018.

21. Dailey AF, Hoots BE, Hall HI, et al. Vital signs: human immunodeficiency virus testing and diagnosis delays_-United States. MMWR Morb Mortal Wkly Rep. 2017;66(47):1300-6. [PubMed: 29190267]

22. Wejnert C, Prejean J, Hoots B, et al. Prevalence of missed opportunities for HIV testing among persons unaware of their infection. JAMA. 2018;319(24):2555-7. [PubMed: 29946714]

23. Centers for Disease Control and Prevention. State HIV testing laws: consent and counseling requirements. https://www.cdc.gov/hiv/policies/law/states/testing.html. Accessed 29 Oct 2018.

24. Bayer R, Philbin M, Remien RH. The end of written informed consent for HIV testing: not with a bang but a whimper. Am J Public Health. 2017;107(8):1259-65. [PubMed: 28640673]

25. Lin X, Dietz PM, Rodriguez V, et al. Routing HIV screening in two health-care settings-New York City and New Orleans, 2011-2013. MMWR Morb Mortal Wkly Rep. 2014;63(25):537-41.

26. Felson UR, Cunningham CO, Zingman BS. Increased HIV testing among hospitalized patients who declined testing in the emergency department. AIDS Care. 2016;28(5):591-7. [PubMed: 26654431]

27. Centers for Disease Control and Prevention. Implementing HIV testing in nonclinical settings: a guide for HIV testing providers. https://www.cdc.gov/hiv/pdf/testing/ CDC_HIV_Implementing_HIV_Testing_in_Nonclinical_Settings.pdf. Accessed 14 Sept 2018.

28. Centers for Disease Control and Prevention. Home tests. https://www.cdc.gov/hiv/testing/ hometests.html. Accessed 14 Sept 2018. 
29. Centers for Disease Control and Prevention. Funding Opportunity Announcement (FOA) PS18-1802: Integrated Human Immunodeficiency Virus (HIV) Surveillance and Prevention Programs for Health Departments. https://www.cdc.gov/hiv/pdf/funding/announcements/ ps18-1802/cdc-hiv-ps18-1802-nofo.pdf. Accessed 14 Sept 2018.

30. National Coalition for the Homeless. HIV/AIDS and homelessness. https:// www.nationalhomeless.org/factsheets/hiv.html. Accessed 10 Dec 2018.

31. Maruschak LM. HIV in prisons, 2001-2010. Washington, DC: Bureau of Justice Statistics; 2015. 


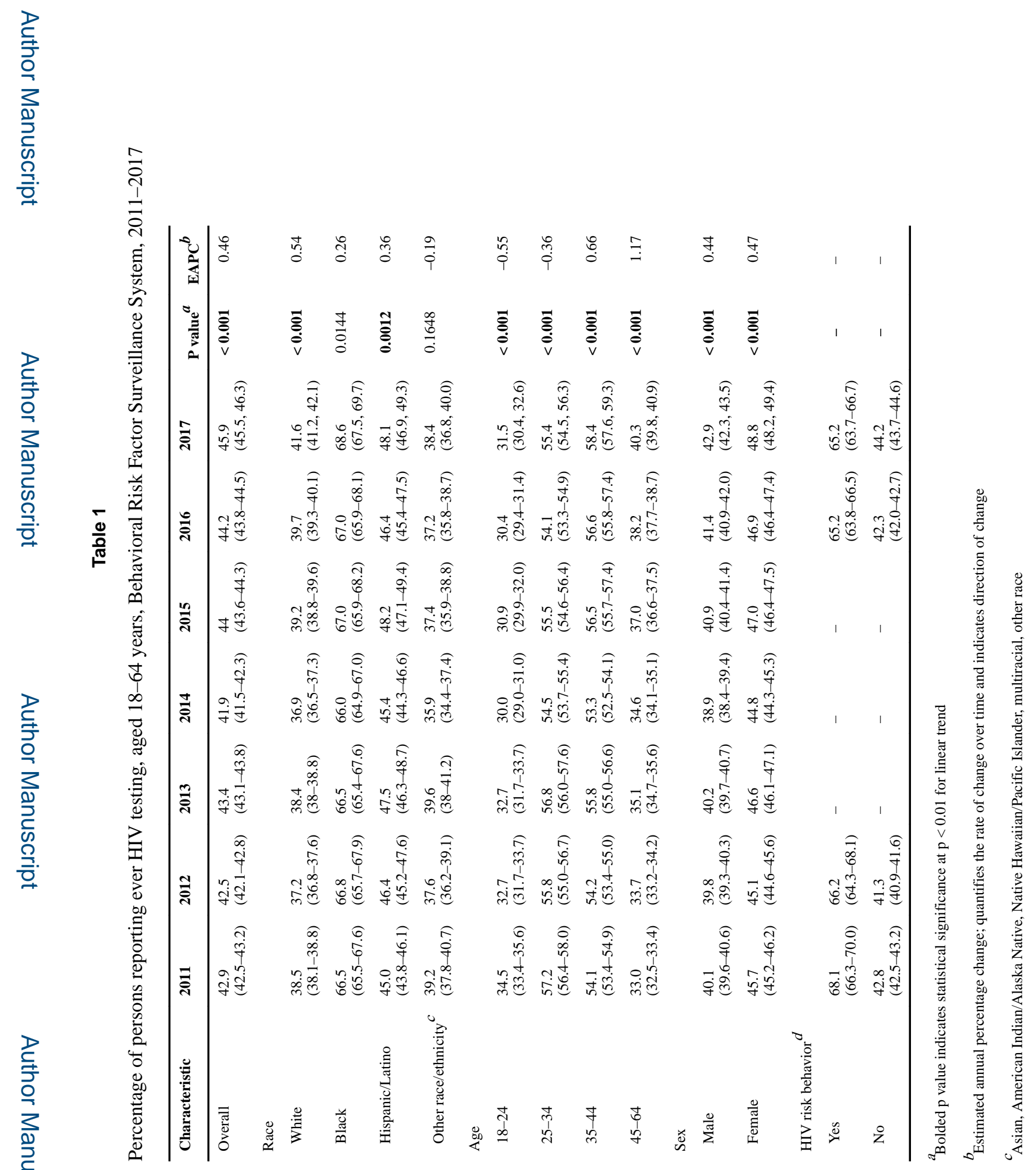




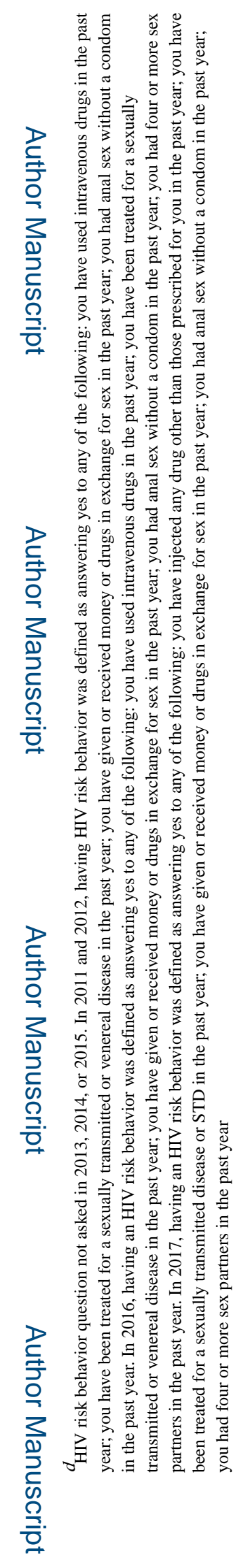

AIDS Behav. Author manuscript; available in PMC 2020 February 01. 


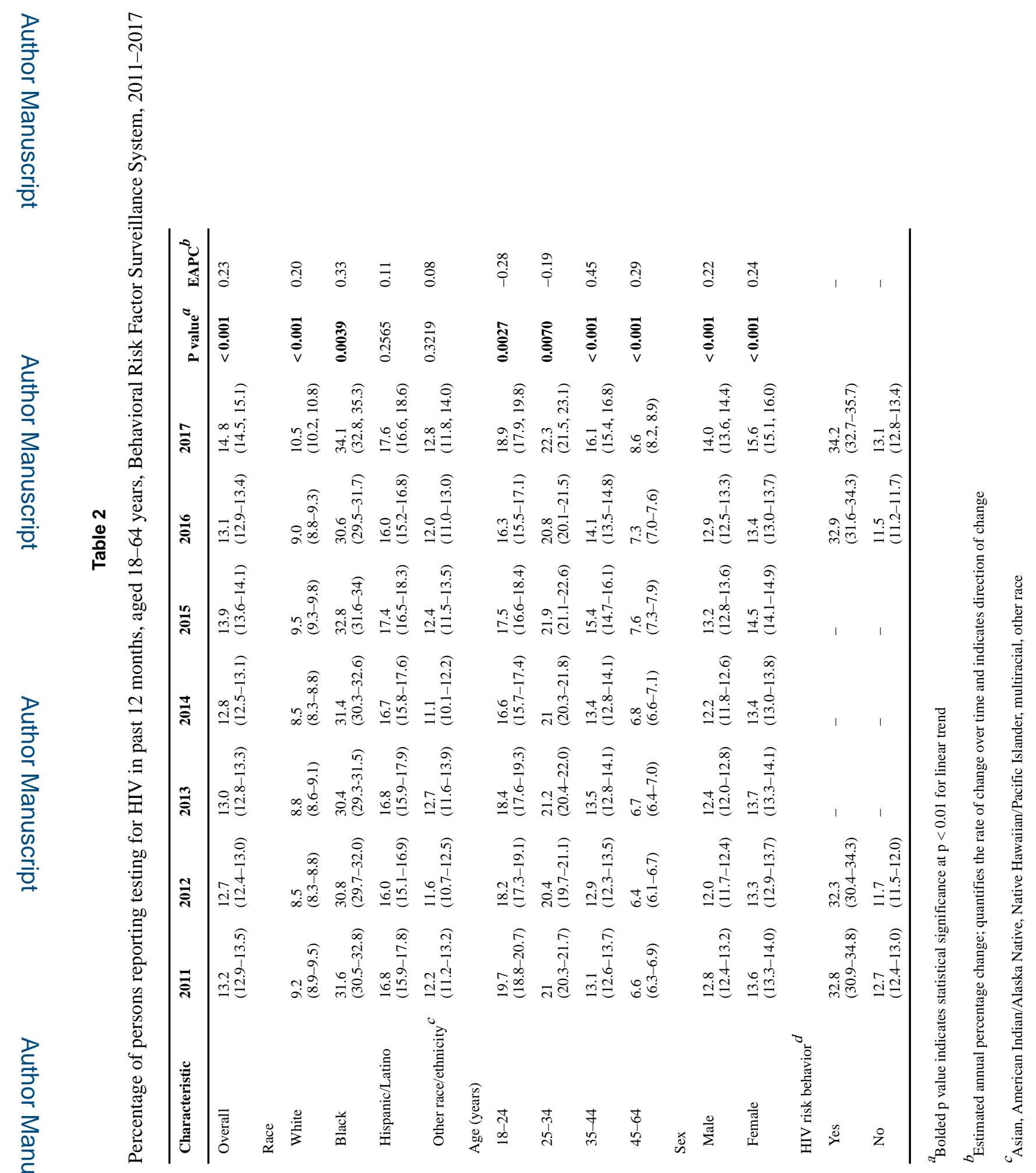




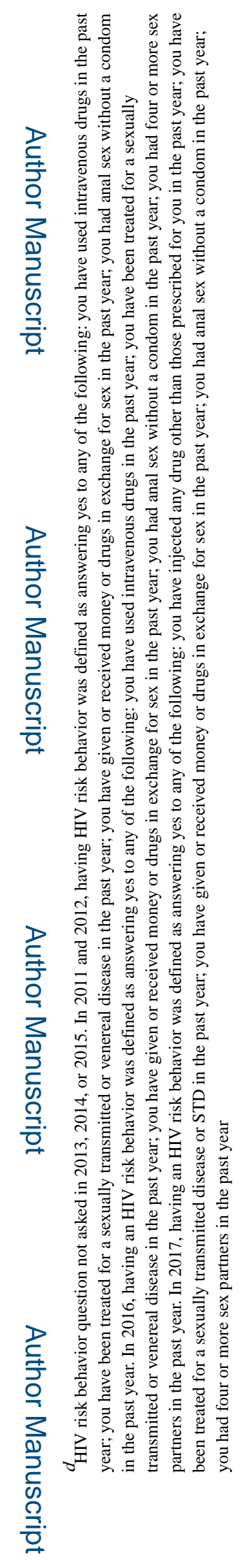

AIDS Behav. Author manuscript; available in PMC 2020 February 01. 